\title{
Practical DHT-Based Location Service for Wireless Mesh Networks
}

\author{
Mehdi Bezahaf, \\ Marcelo Dias de Amorim, and Serge Fdida \\ LIP6/CNRS \\ UPMC Univ Paris 06 \\ Paris, France \\ Mehdi.Bezahaf@lip6.fr, \\ Marcelo.Amorim@lip6.fr, \\ Serge.Fdida@lip6.fr
}

\begin{abstract}
Mobility management in Wireless Mesh Networks (WMN) is a hot topic still lacking a final, widely accepted, and deployed solution. The major drawbacks of existing proposals is the fact that they rely on some sort of flooding-based technique and very often they need support from end-users' devices. Flooding in wireless environments leads to increased latency and broadcast storm problems, thus providing low performances. In this paper, we argue that a DHT-based approach can overcome such limitations and provide an effective solution to the mobility management problem. A preliminary evaluation of our DHT-based scheme on a real WMN test-bed shows encouraging results when compared to previous solutions.
\end{abstract}

\section{Categories and Subject Descriptors}

C.2.1 [Computer-Communication Networks]: Network Architecture and Design-Wireless communication.

\section{General Terms}

Design, Measurement, Experimentation.

\section{Keywords}

Wireless Mesh Network, Mobility Management, Location Service, DHT.

\section{MOTIVATION}

Wireless Mesh Networks (WMNs) are an emerging class of wireless networks, able to organize and configure themselves in a dynamic way [2]. WMNs define two types of nodes: Wireless Mesh Routers (WMRs) and Wireless Mesh Clients (WMCs), where WMRs form a backbone and offer network access to WMCs. Such a two-tier architecture enables new protocol design that leaves out WMCs from the routing, forwarding, and mobility management infrastructure.

Mobility management of WMCs is a very important issue, since users are more and more mobile due to the widespread of wireless technology. As shown in Fig. 1, the architecture of WMNs is placed somewhere between wireless local area

Copyright is held by the author/owner(s).

CoNEXT Student Workshop'09, December 1, 2009, Rome, Italy. ACM 978-1-60558-751-6/09/12.

\author{
Luigi lanonne \\ Technische Universität Berlin, \\ Deutsche Telekom Laboratories \\ Berlin, Germany \\ Luigi@ net.t-labs.tu-berlin.de
}

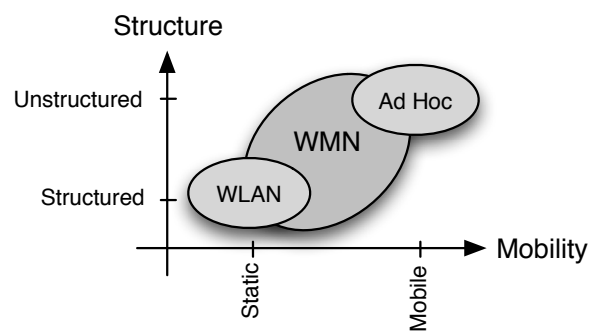

Figure 1: Wireless Networks Taxonomy.

networks (WLAN) and mobile ad hoc networks (MANET). Focusing on the mobility axis, while WMRs are static as access points (APs) in WLANs, WMCs are mobile (as any node in a MANET). The structure axis, instead, shows that WMNs are similar to WLANs, where APs are structured and wired together. WMRs are disposed in a fully distributed and unstructured manner, where each WMR can communicate directly with its direct neighbors in a wireless fashion.

Despite a number of advantages, the performance of mesh networks in terms of mobility management is still poor. This is due to the architectural differences outlined above, making mobility management approaches designed for WLANs and MANETs not suitable for WMNs.

Several mobility management proposals can be found in the literature. They are in general adaptations of existing solutions introduced either for WLANs or for MANETs. This turns out to introduce severe limitations in the design process. Indeed, solutions from the MANET world require that WMCs participate in the mobility management process. In contrast, the main requirement in WMNs is to efficiently detect WMCs' mobility without disrupting existing connections, and with no modifications or additional software at the WMC side. Solutions adapted from the WLAN domain rely on flooding and, while performing reasonably well in the wired part of WLANs, they raise several issues, like the broadcast storm problem [4], in a wireless multi-hop context.

Works like ROAM [5] and EMIPv6 [3] show how Distributed Hash tables (DHT) can be of great help when solving the above mentioned problems. ROAM is based on an overlay Internet infrastructure. It uses rendezvous points on a DHT overlay for indirect data packet delivery, which leads to significant packet-delivery latency. EMIPv6 involves the end users by introducing an end-to-end approach, where 


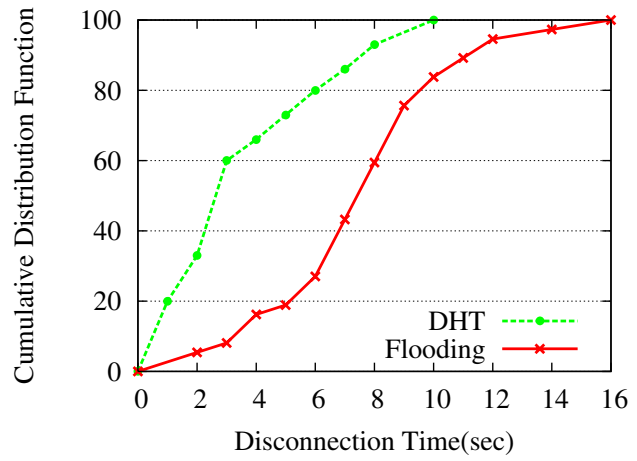

Figure 2: CDF of disconnection time during TCP sender displacement.

nodes exchange directly mobility messages between them. A DHT overlay is used if messages cannot be directly delivered. EMIPv6 requires modifications to the existing IPv6 stack. Moreover, it does not support micro-mobility (i.e., inside the same subnetwork).

We propose a distributed micro-mobility management service for WMNs based on DHT. The use of DHT allows avoiding flooding and retrieving a WMC's position by a simple unicast query.

\section{DHT \& MOBILITY MANAGEMENT}

The above-mentioned limits and issues give us the motivation to explore new mobility management services based on DHTs. The fundamental mechanism behind DHTs consists in mapping objects' identifiers into keys using a hash function, and distributing these keys among nodes of the system. In our context, the DHT is used to manage WMC's localization, mapping WMCs' identifiers into their actual location (i.e., the WMR they are associated with).

By taking into account the WMN's two-tier architecture, our DHT-based approach runs only in the backbone side. In this way, WMCs have no knowledge of the DHT's existence and do not perform any DHT-related operation or localization process (transparency). Our DHT is maintained through underlying routing advertisements concerning topology changes. Therefore, each WMR can contact directly any other WMR. WMRs form a Chord-like logical ring [6], where each WMR owns a slice of the virtual space obtained by hashing the WMRs' identifier. Moreover, each WMR is the locator of all WMCs that fall within its slice; this means that the WMR stores the current position of these WMCs. Thus, through a hash function, WMRs can determine the locator of any WMC in the network, and then the current position of each WMC with a simple unicast query. The proposed approach clearly offers several advantages:

Reduced overhead: Unicast queries generate much less messages on the backbone.

Scalability: WMRs equally share the number of WMCs to manage, allowing the system to work correctly even with large number of WMCs.

Readily deployable: Since our solution is totally transparent to WMCs, it is readily deployable, allowing common wireless devices available in the market to work properly without any upgrade whatsoever.
Table 1: Flooding vs. DHT performance approaches.

\begin{tabular}{|c|c|c|c|}
\hline & Throughput & PDV & \% of lost \\
\hline \hline Flooding & $491 \mathrm{Kbps}$ & $4.417 \mathrm{~ms}$ & $4 \%$ \\
\hline DHT & $505 \mathrm{Kbps}$ & $3.173 \mathrm{~ms}$ & $1.3 \%$ \\
\hline
\end{tabular}

\section{EARLY RESULTS}

We implemented our proposal on a real test-bed [1], on which we performed some preliminary measurements and comparison with a general flooding-based mechanism.

In the first test, we generated UDP traffic during 300 seconds between a mobile UDP client and a UDP server, and observed the impact on the traffic when a client moves and associates with a different WMR. Table 1 summarizes the obtained results for both our DHT and the traditional flooding-based approaches. We observe that the DHT-based approach leads to good performance when compared with the flooding-based approach. The disconnection time using our approach is smaller than the one obtained by the flooding-based solution. As a consequence, both the percentage of packet loss and the packet delay variation (PDV) are lower, while the average throughput is higher.

We performed a similar experiment using TCP traffic. Fig. 2 shows the cumulative distribution function of disconnection time for both approaches. We observe that the disconnection is less than 3 seconds in $60 \%$ of cases using our approach, while it is less than 8 seconds using the floodingbased solution. Moreover, the upper value for the disconnection time is 10 seconds with our approach, while it is equal to 16 seconds with the flooding approach. These improvements are due to the fact that mobility recovery operations in our approach are achieved in unicast fashion instead of flooding, which avoid storm problems and thus packet loss.

\section{CONCLUSION AND FUTURE WORK}

Through a number of practical experiments, we showed that a DHT-based location service is a potential candidate to solve the problem of locating mobile nodes in wireless mesh networks. Next steps of the present work include performing a more thorough and rigorous analysis, using a larger number of nodes and several different metrics. Furthermore, we plan to explore the sensitivity of our proposal to the underlying routing protocol and link variations.

\section{REFERENCES}

[1] Meshdvnet. http://meshdvnet.lip6.fr/.

[2] I. F. Akyildiz, X. Wang, and W. Wang. Wireless mesh networks: a survey. Computer Networks Journal (Elsevier), 47(4):445 487, 2005.

[3] C. Guo, H. Wu, K. Tan, Q. Zhang, J. Song, J. Zhou, C. Huitema, and W. Zhu. End-system-based mobility support in ipv6. IEEE JSAC, 23(11):2104-17, November 2005.

[4] S.-Y. Ni, Y.-C. Tseng, Y.-S. Chen, and J.-P. Sheu. The broadcast storm problem in a mobile ad hoc network. MobiCom '99: The 5th annual ACM/IEEE international conference on Mobile computing and networking, pages 151-162, 1999.

[5] S. Zhuang, K. Lai, I. Stoica, R. Katz, and S. Shenker. Host mobility using an internet indirection infrastructure. ACM Wireless Networks, 11(06):741-56, November 2005.

[6] I. Stoica, R. Morris, D. Karger, M.F. Kaashoek and H. Balakrishnan. Chord: A scalable peer-to-peer lookup service for internet applications. ACM SIGCOMM 2001, August 2001. 